\title{
Brain Abscess and Dental Infections: a review
}

\author{
Johana Maraby-Salgado' ${ }^{1}$, Samer S. Hoz ${ }^{2}$, Alexis Narvaez-Rojas ${ }^{4}$, \\ Guru Dutta Satyarthee ${ }^{5}$, Loraine Quintana-Pajaro ${ }^{1}$, Alfonso \\ Pacheco-Hernandez, Luis Rafael Moscote-Salazar ${ }^{3}$
}

${ }^{1}$ Cartagena University, Cartagena de Indias, COLOMBIA

${ }^{2}$ Department of Neurosurgery, Neurosurgery teaching Hospital-Baghdad, IRAQ

${ }^{3}$ Department of Neurology, Division of Neurosurgery, Cartagena University, Cartagena de Indias, COLOMBIA

${ }^{4}$ Universidad Nacional Autonoma de Nicaragua, MANAGUA

${ }^{5}$ All India Institute of Medical Science, New Delhi, INDIA

\begin{abstract}
Odontogenic infections may cause brain abscesses. Although infrequent, infections can lead to development of aggressive brain lesions that may be lifethreatening for patients. With the advent of new antibiotic treatments, dental abscesses appear to be under control but all patients with high risk of brain abscess should be assessed and treated properly. Hereby, we present an overview of the information available in the literature of the relationship between brain and dental abscesses.
\end{abstract}

Key words: brain abscess; dental infection; neurosurgery

\section{Introduction}

Dental abscess and facial cellulitis put dentists on alert for potentially lifethreatening conditions such as sepsis or airway obstruction, but the risk of a brain abscess is a complication of odontogenic infection that dentists rarely consider. [1] Untreated odontogenic infections can advance to osteomyelitis, cellulitis, myofascial space abscess, lymphadenitis, bacteremia, or sepsis, all of which can be extremely dangerous. Brain abscesses, which are rare, are also a potential type of infection that could arise. These are suppurative infections of the brain parenchyma that are surrounded by a vascularized capsule. In the United States, there are only 1,500 to 2,500 brain abscess cases each year [2,3]. Central nervous system (CNS) infections and their sequelae still constitute a major source of morbidity. $[4,5]$ In the recent past, the introduction of newer broad spectrum antibiotics, improved imaging technology, and intensive care facilities have significantly altered the natural history of CNS infections. [6] Brain abscess (BA) is a universal health problem with a high morbidity and mortality rate; thus, the disease today presents a leading public health 
problem and a major burden on health care facilities all around the world. [6,7] BA is a dynamic focal form of intracranial suppuration and a serious life-threatening emergency. [7] They begin as a localized area of cerebritis and develop into an encapsulated collection of pustular materials presenting as a mass-like lesion, similar to the abscess in other sites. Bacterial brain abscesses have three main etiologies. The most common cause is contiguous spread of infection from the oropharynx, middle ear, and paranasal sinuses. Brain abscesses can also arise from hematogenous dissemination of bacteria. Previous cranial trauma and neurosurgical procedures can also cause brain abscesses. [810] However, in up to $15 \%-30 \%$ of cases, the cause of brain abscesses is unknown. The majority of brain abscesses arise from the direct spread of infection through the facial planes, and often originate in the paranasal sinuses, middle ear and mastoid área [1].

\section{History}

Over the years, there have been a number of case reports of brain abscess, in which the microorganisms were thought to have arisen from a dental source. There are few reports of brain abscesses of odontogenic origin $[11,12]$. Corson et al. [13] reviewed 17 reports from the 1940s to the 1990s, in which the mortality rate was $30 \%(6 / 20)$. Antunes et al. [12] described the location of brain abscesses and their treatment in 11 cases from the 1960s to the 1980s, and in five cases from 2001 through 2007. A systematic review by Moazzam et al. [11] included 60 individual cases, 40 of which were published since 2000 .
They reported the precipitating dental pathology or procedures, the pathogenic microorganisms, the locations of CNS infections, and clinical outcomes [11]. The mortality rate of patients with intracranial bacterial infections of oral origin in that study was improved to $8.3 \%(5 / 60)$ [11]. Ewald et al. [14] reported six cases of pyogenic infection of the CNS secondary to dental infection. They indicated that there was no definable origin for intracerebral or intraspinal infection in up to $25 \%$ of cases, and these are termed "cryptic abscesses" [14], [6]. They noted that a dental focus should always be considered in the evaluation and treatment of "cryptic" CNS infections, and recommended clinical evaluation by a dentist and oral pantomogram [6]. In the report described by Neidert et al. [15] regarding the suspected origin of infection, most cases could be linked to an oral/dental source (23\%) followed by sinusitis (14\%) or a cardiac source $(14 \%)$, and no specific origin of infection could be determined in other cases (43\%). [16]

\section{Mechanism of Infection}

To determine the oral origin of intracranial infections, oral surgeons should know the underlying mechanism of oral bacterial spread into the CNS. Hematogenous spread via bacteremia is probably a more common route tan direct venous drainage. Therefore, there appears to be no predilection for the location of odontogenic foci. There may be no apparent past history or symptoms of acute inflammation caused by odontogenic foci or dental procedures prior to 
development of brain abscess. Microbes can spread as a result of acuteinfection with a large number of involved pathogens or highly virulent microorganisms, as well as chronic infection with recurrent bacteremia [16].

The oral cavity is well recognised as being home to a rich and abundant microflora. [13] In particular, dental plaque contains one of the most concentrated accumulations of microorganisms in the human body, with approximately 350 different bacterial strains isolated in marginal periodontitis and 150 in endodontic infections. Bacteria gaining access to the blood stream may then spread to distant sites and there has long been an association between oral bacteria, in particular a-haemolytic streptococci, and bacterial endocarditis. Dental procedures alleged to have caused a brain abscess are very wide ranging, and include extraction, operative dentistry, periodontal therapy, dental local anaesthetic injection and dental prophylaxis [17]. This would suggest that the mechanism esponsible for the production of the bacteraemia is not as critical as the host response. In this case report there was no apparent history of an acute dental infection or dental procedure prior to development of the brain abscess. However it would appear that from very mild manipulations of the mouth, bacteria can transfer into the blood stream. One study in children found a transient bacteraemia in $38.5 \%$ of episodes of tooth brushing, while procedures such as dental extraction, produce a massive shower of organisms into the blood stream [18]. Within $1 \mathrm{~min}$ of a dental procedure, microorganisms may have reached the heart, lungs and peripheral capillary system. In most individuals the reticuloendothelial system eliminates these microbes in a matter of minute [13]. This is not the case in immunocompromised populations, for example, organ transplant patients and those with AIDS where there has been a reported increased incidence of brain absces. [6] Although certain underlying brain pathologies such as a previous stroke [13] or underlying neoplasm [13] may serve as a nidus for abscess formation, in most cases there is no apparent predisposing brain lesion.

\section{Epidemiology}

Brain abcess (BA) is one of the most serious diseases of the CNS. This condition is more common among men twice to three times, and morbidity rate is highest in fourth decade of the life [3,25] Brain abcess is still associated with high morbidity, including seizures (up to $80 \%$ ), persistent altered mental status, and focal motor deficits. [26] Brain abscess continues to be a significant problem in the developing world due to large scale poverty, illiteracy, and lack of higiene.

A BA may be developed from three sources and one of these is through from pericranial contiguous focus in $25-50 \%$ of cases (such as the sinuses, middle ear, or dental infection), interestingly dental infections, ethmoid or frontal sinusitis (usually spreads to the frontal lobe), and subacute or chronic otitis media or mastoiditis (preferentially spreads to the inferior temporal lobe and cerebellum). [4] 


\section{Etiology}

BA are frequently polymicrobial, From oral cavity, hemathogenous spread (intraabdominal/ pelvic infections), and from otorhinolaringeal infections the commonest organisms isolated are anaerobic pathogens (Streptococci, Bacteroides spp., Prevotella melaninogenica, Propionibacterium, Fusobacterium, and Actinomyces and aerobic gram-negative rods, like Morganella morganii) [4,19-21]

There are reports that E. faecalis was also found in a case of brain abscess secondary to odontogenic procedures in a patient with teleangiectasia, although its occurrence is rare and infrequent, since it is usually a nosocomial pathogen [22] E. faecalis is an infrequent causative agent of brain abscess. This organism is usually a nosocomial pathogen, and its presence in the central nervous system (CNS), rarely if ever as a cause of meningitis, has been associated with anatomic defects, prior neurosurgery and trauma, or high-grade bacteremia and immunodeficiency [22,23]. Common portals of entry for enterococcal bacteremia are the urinary tract and sites of intra-abdominal and pelvic sepsis [22-24]. However, recently, literature provided evidence that due to its ability to produce biofilms, E. faecalis is often involved in endodontic infections [23], rendering sites of dental work a potential portal of enterococcal bacteremia. [22] A literature review revealed four cases of brain abscess involving pus from which $E$. faecalis was isolated $[22,25,26]$
There have also been reports of Aggregatibacter actinomycetemcomitans, which is recognised as one of the major pathogens in destructive periodontal disease. A cerebral abscess linked to a dental source is a rare occurrence, since in most individuals the blood-brain barrier, along with the immune response, will exclude bacteria. [27] A. actinomycetemcomitans has been also described as a cause of meningitis, brain abscess, endophthalmitis (with and without concomitant endocarditis), soft tissue infections, septic arthritis, osteomyelitis, and endocarditis. It may be part of the endogenous flora of the mouth and can be recovered from about $20 \%$ of healthy teenagers and adults It is normally found in dental plaque, periodontal pockets and gingival sulcus, and is one of the major pathogens in adult and juvenile forms of periodontitis. It is present in more than $50 \%$ of adults with refractory periodontitis and $90 \%$ of patients with localised aggressive periodontitis. [27, 28]

\section{Clinical presentation}

BA can be primarily present in four basic syndromes, viz. focal mass expansion, intracranial hypertension, diffuse destruction, and focal neurological deficit. [29] In most cases, predisposing risk factors, such as congenital heart disease, decreased immunity, or the presence of a septic focus, can be identified. [4]

The clinical presentation of intracranial abscess is dependent on the origin of infection, site, size, number of lesions, specific brain structures involved, the neighborhood 
anatomy disturbances involving cisterns, ventricles, and the dural venous sinuses, and any secondary cerebral injury. [30] Frequently patients present with symptoms of increased intracranial pressure (headache, nausea/vomiting, and altered mental status), focal neurologic deficits, and fever (although fever can be absent in 30-76\% of cases). Nathoo et al. [7] reported that headaches, fever, and nuchal rigidity were the commonest clinical presentation. The duration of symptoms ranged from 1 day to 8 weeks (average $11.4 \pm 10$ days; $\mathrm{P}=0.7$ ). [4] This is generally present in patients with brain abscess, secondary to periodontal infections.

\section{Diagnosis}

Neuroimaging, usually a computed tomography (CT) scan with contrast, is essential to diagnose a BA. The typical finding on CT scan or MRI is a hypodense lesion with a contrast-enhancing ring. [26] CT facilitates early detection, exact localization, and accurate characterization, determination of number, size and staging of the abscess,

Diagnosing the microbiological source of any infection must be based on sound microbiological methodology. Bacteria isolated from the distant abscess must represent that found within the oral cavity. An array of molecular fingerprinting techniques based upon nucleotide sequencing can now be applied to provide supportive evidence for the identification of isolates from different sources. Such techniques should be utilized to further our understanding as to whether oral sources of infection can give rise to brain abscess. [13]

The differential diagnosis between BA and brain tumors is of extreme importance. Rahmat-Langendoen et al. explores difficult.their similarity and shows that the symptoms are similar that makes the diagnosis of BA of dental origin extremely. [28]

\section{Treatment}

Recently, Mouren et al, described in a case report a patient with brain abscess secondary to odontogenic injection using metronidazole, vancomycin, and cefotaxime [31]. Although BA is essentially a surgical pathology, Arlotti et al. [32] recommend that choice of patients for a medical approach must be made on an individual basis. These authors consider best candidates for medical treatment to be those with a small abscess $(<2.5 \mathrm{~cm})$, in good initial

clinical condition, Glasgow Coma Scale (GCS) > 12, and for whom the etiology is well -known (microorganism isolated from material other than the abscess pus) recommendation grade $\mathrm{C}$; or in the case of multiple abscesses, after surgery of abscesses $>2.5 \mathrm{~cm}$ or surgery of abscesses that cause a mass effect, or in patients at serious risk of operation even if in these, the final decision must consider that the prognosis is often bad in any case recommendation grade D. [4]

For Muzumdar et al. [29], there are no pragmatic rules for treatment of brain abscess and each case must be individualized and treated on its own merits. The main stay of the treatment includes prompt action and 
institution of antibiotic therapy. Penicillin and chloramphenicol have long been used until we have opted for cefotaxime/ ceftriaxone/ceftazidime, vancomycin, and metronidazole, and this is in agreement with the treatment administered to the patient. Steroid administration should be generally avoided unless the patient demonstrates signs of meningitis or disproportionate cytotoxic edema posing a life threatening problem. Legg advocated anticonvulsant therapy for 5 years to all patients with cerebral abscess. Discontinuation of antiepileptic drugs can be considered when patient is seizure-free for at least 2 years after surgery and electroencephalogram. [29]

Bacteroides, Peptostreptococcus, and Fusobactrium are common anaerobes and are sensitive to metronidazole. Staphylococcus is common in post-traumatic and postoperative cases; in infants and neonates, postmeningitic abscess is caused by gramnegative organisms. [17] Sulpha drugs are most effective in Nocardia and vancomycin against Staphylococcus. [4]

\section{Conclusions}

A cerebral abscess linked to a dental source is a rare occurrence and its clinical features are often nonspecific. It is worth noting that this latter component calls upon dental clinicians to pay more attention in the identification and treatment of oral and odontogenic sources for brain abscesses.

\section{Correspondence}

Dr. Luis Rafael Moscote-Salazar

e-mail:mineurocirujano@aol.com

\section{References}

1. Christine E. Hibberd TDN. Brain Abscess Secondary to a Dental Infection in an 11-Year-Old Child: Case Report. J Can Dent Assoc 2012;78:49.

2. Sung Yong Park DWS, Chul Min Park, Min Seok Oh, Dong-Kun Lee. Brain abscess due to odontogenic infection: a case report. J Korean Assoc Oral Maxillofac 2014;40:147-51.

3. Mamelak AN MT, Obana WG, Rosenblum ML. Improved management of multiple brain abscesses: a combined surgical and medical approach. Neurosurgery 1995;36:76-85.

4. Alvis-Miranda H, Castellar-Leones S, Elzain M, Moscote-Salazar L. Brain abscess: Current management. Journal of Neurosciences in Rural Practice. 2013;4(5):67. 5. Muzumdar D. Central nervous system infections and the neurosurgeon: A perspective. International Journal of Surgery. 2011;9(2):113-116.

6. Mathisen GE JJ. Brain abscess. Clin Infect Dis. 1997;25:763- 79.

7. Nathoo N NS, Narotam PK, Van Dellen JR. . Brain Abscess: Management and Outcome Analysis of a Computed Tomography Era Experience with 973 Patients. World Neurosurg 2011;75:716-26.

8. Xiao F TM, Teng LJ, Tseng HM, Tsai JC. Brain abscess: clinical experience and analysis of prognostic factors. Surg Neurol 2005;63:442-9.

9. Tseng JH TM. Brain abscess in 142 patients: factors influencing outcome and mortality. . Surg Neurol 2006;65:557-62.

10. Tonon E SP, Gallucci M, Vaglia A. Brain abscess: clinical aspects of 100 patients.. Int J Infect Dis 2006;10:103-9.

11. Moazzam AA RS, Sedghizadeh PP, Zada G, Habibian M. Intracranial bacterial infections of oral origin. J Clin Neurosci 2015.

12. Antunes AA dSS, de Carvalho RW, Avelar RL, Pereira CU, Pereira JC Brain abscess of odontogenic origin. J Craniofac Surg. 2011;22:2363-5.

13. MA Corson KP, RA Seymour. Are dental infections a cause of brain abscess? Case report and review of the literature. Oral Diseases 2001;7:61-5.

14. Ewald C KS, Kalff R Pyogenic infections of the central nervous system secondary to dental affections-a report of six cases. Neurosurg Rev 29:. 2006;29:163-6 (discussion 6-7). 
15. Neidert MC KK, Actor B, Regli L, Bozinov O, Burkhardt JK Preoperative C-reactive protein predicts the need for repeated intracerebral brain abscess drainage. . Clin Neurol Neurosurg 2015;131:26-30.

16. Masaya Akashi KT, Junya Kusumoto, Shungo Furudoi, Kohkichi Hosoda, Takahide Komori. Brain Abscess Potentially Resulting from Odontogenic Focus: Report of Three Cases and a Literature Review. J Maxillofac Oral Surg. 2016.

17. Schuman NJ TJ. Brain abscess and dentistry: a review of the literature. Quintessence Int. 1994;25:411-3.

18. Roberts GJ HH, Sury MRJ et al Dental bacteraemia in children. Paediat Cardiol 1997;18:24-7.

19. W. M. Pyogenic infective diseases of the brain and spinal cord. 1893.

20. Beller AJ SA, Praiss I. Brain abscess.Review of 89 cases over a period of 30 years. J Neurol Neurosurg Psychiatry 1973;36:757-68.

21. Bhatia R TP, Banerji AK. Brain abscess-an analysis of 55 cases. Int Surg 1973;58(565-8).

22. Eleni Mylona CV, Vasilios Papastamopoulos and Athanasios Skoutelis. Brain Abscess Caused by Enterococcus faecalis following a Dental Procedure in a Patient with Hereditary Hemorrhagic Telangiectasia. J Clin Microbiol 2012;50(5):1807.

23. Moellering R, Jr. Enterococcus species, Enterocuccus bovis and Leuconstoc species. Principles and practice of infectious diseases 2010.

24. Fisher K PC. The ecology, epidemiology and virulence of Enterococcus. Microbiology 2009;155:1749 -57 .
25. Inamasu J ea. Brain abscess developing at the site of preceding intracerebral hemorrhage. J Neurol 2002;249:221-3.

26. Siatouni A ea. Brain abscess following intracerebral haemorrhage. J Clin Neurosci 2007. ;14:986 -9.

27. Brady P, Bergin, Sarah, Cryan, Bartley, Flanagan, Oisin. Intracranial abscess secondary to dental infection. Journal of the Irish Dental Association. 2014;60 (1):32-4. 28. Marcelo Rodrigues Azenha GH, Idelmo Rangel Garcia Jr. Multiple brain abscess from dental origin: case report and literature review. Oral Maxillofac Surg 2012;16:393-7.

29. Muzumdar D JS, Goel A. Brain abscess: An overview. Int J Surg 2011;9:136-44.

30. Arlotti M GP, Pea F, Tomei G, Vullo V, De Rosa FG, et al. Consensus document on controversial issues for the treatment of infections of the central nervous system: Bacterial brain abscesses. Int J Infect Dis 2010;14(4):7992.

31. Marouene Ben Hadj Hassine LO, Amine Derbel, Nabiha Douki. Cerebral Abscess Potentially of Odontogenic Origin. Case Reports in Dentistry. 2015;1:1-4.

32. Arlotti M GP, Pea F, Tomei G, Vullo V, De Rosa FG, et al. Consensus document on controversial issues for the treatment of infections of the central nervous system: Bacterial brain abscesses. Int J Infect Dis. 2010;14(4):7992. 